\title{
The Evolving Role of Local Treatments for HCC in the Third Millennium
}

\author{
PAOLO MAGISTRI ${ }^{1,2}$, GIUSEPPE TARANTINO ${ }^{1}$, ROBERTO BALLARIN $^{1}$, MASSIMILIANO BERRETTA $^{3}$, \\ ANNARITA PECCHI ${ }^{4}$, GIOVANNI RAMACCIATO ${ }^{2}$ and FABRIZIO DI BENEDETTO ${ }^{1}$ \\ ${ }^{1}$ Hepato-Pancreato-Biliary Surgery and Liver Transplantation Unit, \\ University of Modena and Reggio Emilia, Modena, Italy; \\ ${ }^{2}$ Department of Medical and Surgical Sciences and Translational Medicine, \\ Sapienza - University of Rome, Rome, Italy, \\ ${ }^{3}$ Department of Medical Oncology A, National Cancer Institute of Aviano, Aviano, Italy; \\ ${ }^{4}$ Department of Radiology, University of Modena and Reggio Emilia, Modena, Italy
}

\begin{abstract}
Background: Hepatocellular carcinoma (HCC) represents the fifth most common malignancy and the third cancer-related cause of death worldwide. The aim of this review was to clarify the role of local treatments for $\mathrm{HCC}$, analyzing the indications and defining future perspectives. Materials and Methods: A systematic literature search was performed independently by two of the authors according to the PRISMA statement guidelines. The search was limited to studies reported in English between January 2005 and June 2016. Results: The literature search yielded 238 articles; after duplicates were removed, 179 titles and abstracts were reviewed. Most relevant data and articles about radiofrequency ablation, transarterial chemoembolization, percutaneous ethanol injection, microwave ablation and radioembolization are reported and discussed. Conclusion: Data in the literature are confusing and difficult to compare due to the lack of prospective studies. Multidisciplinary and tailored approaches for each patient are key features, considering both guideline indications and patient-specific characteristics, and enhance hospital-specific best practice.
\end{abstract}

This article is freely accessible online.

Correspondence to: Professor Fabrizio Di Benedetto, HepatoPancreato-Biliary Surgery and Liver Transplantation Unit, University of Modena and Reggio Emilia, Via del Pozzo 71, 41124, Modena (MO), Italy. Tel.: +39 0594224740, Fax: +39 0594223765, e-mail: fabrizio.dibenedetto@unimore.it

Key Words: Hepatocellular carcinoma, local treatments, TACE, thermo-ablation, PEI, microwave, yttrium-90, review.
Despite the advances in oncological and surgical treatments, hepatocellular carcinoma (HCC) is still a global burden, representing the fifth most common malignancy and the third cancer-related cause of death worldwide (1). In the US, the incidence of $\mathrm{HCC}$ has almost tripled in the last 30 years, and it is the fastest rising cause of cancer-related deaths (2). HCC prevalence varies worldwide: the highest incidence is registered in Asia (>20 cases/100,000) than in North America and Europe $(<5$ cases/100,000) (1). Interestingly, the analysis of the Italian Liver Cancer database by Santi et al. published in 2012 showed significantly better survival for patients with HCC and cirrhosis recruited more recently compared to their counterparts recruited 20 years earlier. However, this trend was no longer confirmed over the subsequent 10 years (3). HCC outcomes have improved over time in Italy, mainly due to wider application of surveillance and increased propensity to treat patients (4). Therefore, early diagnosis represents a crucial step in the global approach to HCC.

For a better understanding of the screening and surveillance protocols, we first need to reiterate the risk factors and causes of HCC. In 70-90\% of cases, HCC develops in patients with chronic liver disease, and risk factors depend on the region where the studies are conducted. For example, while hepatitis $\mathrm{C}$ virus (HCV) infection is a major factor in Europe, Japan and North America accounting for $50 \%$ to $70 \%$ of cases, HBV is responsible for $10-15 \%$, alcohol for $20 \%$ and other causes for $10 \%$. Conversely, in Asia and Africa, HBV is associated with $70 \%$ of cases and HCV with $20 \%$. Depending on the study, the relative risk of developing a tumor is close to 100fold in HBV carriers versus non-carriers, while the incidence of HCC in individuals with cirrhosis due to $\mathrm{HCV}$ is $3-5 \%$ per year (1). The comorbidities of each patient can modify the risk of developing an $\mathrm{HCC}$ in the presence of cirrhotic 
degeneration of the liver. The highest 5 -year cumulative risks are seen in those with $\mathrm{HCV}$-associated cirrhosis $(17 \%$ in the West and 30\% in Japan), hemochromatosis (21\%), HBVassociated cirrhosis (10\% in the West and $15 \%$ in Asia), alcoholic cirrhosis (8-12\%), and biliary cirrhosis (4\%) (2). Alcohol abuse is a well-known cause of chronic liver disease and cirrhosis. Heavy alcohol intake is defined as the ingestion of more than 50-70 g per day for prolonged periods (2). In addition, the association between the increased risk of HCC in heavy alcohol drinkers and genetic polymorphisms of the enzymes participating in the metabolic pathway of ethanol, and in particular the frequency of aldehyde dehydrogenase 2 $(A L D H 2)$ genotype polymorphism, has been proposed as a mechanism by which HCC develops (5). Among non-viralrelated $\mathrm{HCC}$ cases, hereditary hemochromatosis confers an elevated risk. As shown in literature, patients diagnosed with hereditary hemochromatosis who died were 23 -fold more likely to have liver cancer compared to those without a diagnosis of hemochromatosis (5). Metabolic syndrome also plays a pivotal role: non-alcoholic fatty liver disease (NAFLD), the liver manifestation of metabolic syndrome, has become the most common liver disease in the Western world and its progression results in cirrhosis $(6,7)$. In fact, NAFLD confers both additive and independent risk to the development of HCC: $65 \%$ of HCCs developing in patients with metabolic syndrome occurred in livers with mild fibrosis, compared to $20 \%$ in those with other causes of HCC. However, once cirrhosis is established in NAFLD, the risk of HCC may be similar to that seen in cirrhosis due to HCV (6). Although the association between increasing body mass index (BMI) and $\mathrm{HCC}$ is much stronger in individuals with concomitant $\mathrm{HCV}$ infection than in persons with HBV infection (2), recent studies demonstrated a significantly increased risk of HCC among obese [odd ratio $(\mathrm{OR})=3.5,95 \%$ confidence Interval $(\mathrm{CI})=1.3-9.2]$ or diabetic $(\mathrm{OR}=3.5,95 \% \mathrm{CI}=1.6-7.7)$ patients without viral hepatitis, and a high prevalence in cryptogenic cirrhotic patients (5). As a matter of fact, the association between BMI and liver cancer is independent of geographic location, alcohol consumption, and history of diabetes (2). Lastly, aflatoxin B1 exposure, tobacco use, coffee consumption, use of oral contraceptives and betel quid are other proven risk factors of $\mathrm{HCC}(2,5)$. Of note, HIV-positive patients with HCC had a significantly shorter survival time than HIV-negative counterparts (8).

The comprehension of HCC development is made even harder when considering how many molecular pathways and genetic alterations are involved. p53/RB, WNT/ $\beta$-catenin, PI3K/PTEN/AKT/mTOR and Hedgehog are the pathways most well-known to play an important role in the development and progression of HCC (9). Moreover, it was demonstrated that $\mathrm{HBx}$, a multifunctional protein encoded by the HBV genome, plays a pro-carcinogenic role, since it has the ability for the transcriptional transactivation and can activate the JAK/STAT signaling pathway but impair the p53 function (9). It has been demonstrated that $90 \%$ of human HCCs have increased telomerase expression. The mechanisms of telomerase reactivation are mutually exclusive and include telomerase reverse transcriptase (TERT) promoter mutations (54-60\%), TERT amplification (5-6\%), and HBV insertion into the TERT promoter (10$15 \%)$ (10). Tumor-stromal communication and epithelial to mesenchyme transition are also important factors participating in tumor progression (11-13). Interestingly, long non-coding RNAs also seem to be strongly associated with liver cancer, with a potential role as biomarkers for disease diagnosis, prognosis, or therapeutic response, as well as being direct targets for therapeutic intervention (14).

Both Western (American Association for the Study of Liver Diseases and European Association for the Study of the Liver) and Eastern (Asian Pacific Association for the Study of the Liver and Japan Society of Hepatology) guidelines are available for HCC surveillance and screening. Screening is recommended in patients with: (i) cirrhosis of any etiology, with conserved liver function [Child-Turcotte-Pugh (CTP) class $\mathrm{A}$ and $\mathrm{B}$ ], lacking severe comorbidities; (ii) decompensated cirrhosis (CTP C) on a transplant waiting list; (iii) non-cirrhotic chronic $\mathrm{HBV}$ infection with active hepatitis or a family history of HCC; and iv) non-cirrhotic HCV infection and advanced liver fibrosis (F3) (1). Recently, surveillance ultrasound imaging of the liver every 6 months has increased reliability versus the use of serum levels of alpha fetoprotein (AFP), the classical HCC marker, due to poor sensitivity of AFP $(6,15)$. Other markers such as glypican-3, heat-shock protein 70 and SAL-like protein 4 are promising but currently not validated $(9,11)$. Computed tomography (CT) can be proposed for screening in obese patients due to technical issues on ultrasound performance (6). However, CT is always indicated when a lesion is suspected on ultrasound: intense arterial uptake, followed by washout of contrast in the venous phase, is the hallmark radiological sign of HCC (15). According to the most recent trends, nodules $<1 \mathrm{~cm}$ do not correspond to an HCC in most cases, and even when they do, confident diagnosis is currently almost impossible to be achieved. Follow-up with ultrasound scan of those small nodules should be performed over time until they grow beyond $1 \mathrm{~cm}$ or vanish (16). This is because biopsy of such small lesions in patients with cirrhosis is not completely reliable: sampling errors and difficulty in distinguish well-differentiated HCC from dysplastic nodules must always be considered, making it impossible to rule out malignancy on the basis of a negative biopsy (16). While Eastern societies always include contrast enhanced ultrasound (CEUS) in the diagnostic pathway, Western societies do not consider CEUS an appropriate study in the diagnostic approach to HCC, due to the theoretical difficulty in differentiating $\mathrm{HCC}$ from cholangiocarcinoma (1). 
Several classification and staging systems have been proposed for the management of HCC. As a matter of fact, such a variety hides the latent difficulty in adopting a really functional algorithm. CTP (17), Model for Endstage Liver Disease (MELD) (18), TNM classification (19), tumor volume estimation (20), and evaluation of the patient's Eastern Cooperative Cancer Group (ECOG) performance status (21), are well-known systems but the most used is the Barcelona Clinic Liver Cancer staging system (BCLC) (22). BCLC is a multidimensional platform based on patient's performance status, liver function (calculated using the CTP score) and tumor dimensions that stratifies HCC severity and consequent therapeutic approaches into five categories. According to that system, very early (BCLC 0 ) and early stages (BCLC A) are amenable to curative treatments, such as tumor resection, liver transplantation and tumor ablation. In the first category, radical treatments can completely eradicate the tumor, while in the second, the survival does not exceed 3 years if HCC is not treated. On the other hand, intermediate-stage (BCLC B) HCC can benefit from transarterial chemoembolization (TACE), improving median survival to more than 24 months compared to 16 months without treatment. Advanced-stage (BCLC C) HCC can be treated with Sorafenib: it was the only therapy that demonstrated some survival benefit, compared to the average survival of 8 months for patients at this stage without any treatment. Lastly, terminal stage (BCLC D) HCC requires only supportive care (1). However, given its heterogeneity and the intricate background of HCC development, the therapeutic approach should always be multidisciplinary and patienttailored in order to offer the best treatment possible (23, 24). Notably, the BCLC system is not rigid and should be used with the consideration that a patient being evaluated for therapy may move from the indication corresponding to an early stage to that of intermediate or advanced stage because of a specific patient profile that may contraindicate the initially optimal approach, reflecting the 'treatment stage migration' concept (16). This demonstrates the importance of developing optimal downstaging strategies to make patients move backwards to 'earlier' stages that can benefit from therapeutic approaches. In fact, although there are no randomized trials assessing the benefit of treatment of patients on the transplant waiting list, it seems reasonable and is a common practice to treat patients either with percutaneous ablation or TACE to prevent progression and bridge patients to liver transplantation when the waiting time is longer than 6 months (16).

The aim of this review was to clarify the role of local treatments for HCC, analyzing the current indications and defining future perspectives.

\section{Materials and Methods}

Literature search. Preferred Reporting Items for Systematic Reviews and Meta-Analyses (PRISMA) statement guidelines for conducting and reporting systematic reviews were followed (25).

A systematic literature search was performed independently by two of the Authors (PM and GT) using PubMed, EMBASE, Scopus and the Cochrane Library Central. The search was limited to studies in humans and to those reported in the English language between January 2005 and June 2016. No restrictions were set for the type of publication. Participants of any age and sex who underwent local treatment for HCC were included in this study.

The following MeSH search headings were used: "hepatocellular carcinoma" OR "HCC" OR "hepatoma" AND "TACE" OR "radiofrequency ablation" OR "PEI" OR "microwave" OR "radioembolization" OR "local treatment". Extensive crosschecking of the reference lists of all retrieved articles that fulfilled the inclusion criteria further broadened the search. For all of the databases, the last search was run on June 1st, 2016.

Study selection. The same two Authors screened the titles and abstracts of the primary studies that were identified in the electronic search. The following criteria were adopted for inclusion in this review: (i) Studies comparing the outcomes of different local treatments; (ii) studies reporting at least one perioperative outcome; and (iii) if more than one study was reported by the same institute, only the most recent or the highest quality study was included.

The following exclusion criteria were set: (i) Original studies assessing the outcome of surgical approach alone; (ii) review articles, letters, comments and case reports; and (iii) studies in which it was impossible to retrieve or calculate data of interest.

The literature search yielded 238 articles; after duplicates were removed, 179 titles and abstracts were reviewed. The most relevant data and articles are reported in the results section and later discussed.

Data extraction. The main data were extracted as follows: (i) First author, year of publication and study type; (ii) number and characteristics of patients and (iii) treatment outcomes including hospital stay, mortality rate, recurrence rate, 5-year overall survival and 5-year disease-free survival. All relevant texts, tables and figures were reviewed for data extraction and whenever further information was required, the corresponding authors of the papers were contacted by e-mail.

Discrepancies between the two reviewers were resolved by consensus discussion or with the opinion of the senior author (FDB). Results are summarized in Tables I-III.

\section{Results}

Ablative therapies: Radiofrequency ablation (RFA) percutaneous ethanol injection (PEI) - microwave. HCC can be treated with RFA in several conditions. In 2015, Kim and colleagues reported their experience in a retrospective study of 604 consecutive patients affected by small HCCs $(<3 \mathrm{~cm})$ and treated between 2000 and 2009 with either surgery (273 patients) or RFA (331 patients) (26). Inclusion criteria were: (i) well preserved liver function of CTP class A; (ii) normal serum bilirubin level (less than $1.5 \mathrm{mg} / \mathrm{dl}$ ); and (iii) no 
Table I. Outcomes of the most relevant articles on tumor ablation.

\begin{tabular}{|c|c|c|c|c|c|c|}
\hline Author (Ref) & Year & No. of patients & Type of study & Study interval & Study design & Major outcomes \\
\hline Signoriello et al. (29) & 2012 & 425 & $\mathrm{R} / \mathrm{M}$ & $1998-2002$ & $\begin{array}{l}\text { Patients treated with local } \\
\text { approach were compared } \\
\text { to evaluate overall } \\
\text { survival with a median } \\
\text { follow-up of } 7.7 \text {. years }\end{array}$ & $\begin{array}{c}\text { No statistically significant } \\
\text { difference comparing RFA } \\
\text { versus } \text { PEI, surgery versus } \\
\text { TACE/TAE, and RFA/PEI } \\
\text { versus } \text { TACE/TAE }\end{array}$ \\
\hline Chang et al. (28) & 2012 & 39 & $\mathrm{R} / \mathrm{S}$ & $2002-2007$ & $\begin{array}{l}\text { Patients with incompletely } \\
\text { treated single HCC } \\
(\leq 5 \mathrm{~cm}) \text { after TACE } \\
\text { were treated with RFA }\end{array}$ & $\begin{array}{l}\text { Local recurrence-free and } \\
\text { survival rates are comparable } \\
\text { as demonstrated in previous } \\
\text { studies of RF ablation alone }\end{array}$ \\
\hline Lin et al. (31) & 2013 & 1036 & $\mathrm{R} / \mathrm{M}$ & 2004-2006 & $\begin{array}{l}\text { Patients receiving PEI or } \\
\text { RFA as first-line treatment for } \\
\text { newly-diagnosed stage I-II } \\
\text { HCC were enrolled }\end{array}$ & $\begin{array}{l}\text { RFA is related to better } \\
\text { OS compared to PEI } \\
\text { regardless of tumor size }\end{array}$ \\
\hline Zhang et al. (32) & 2013 & 155 & $\mathrm{R} / \mathrm{S}$ & 2006 & $\begin{array}{l}\text { Patients receiving PEI or } \\
\text { RFA as first-line treatment for } \\
\text { newly-diagnosed stage I-II } \\
\text { HCC were enrolled }\end{array}$ & $\begin{array}{l}\text { RF ablation and MW ablation } \\
\text { are both effective methods } \\
\text { in treating hepatocellular } \\
\text { carcinomas, with no significant } \\
\text { differences in CA, LTP, DR, and OS }\end{array}$ \\
\hline Hasegawa et al. (30) & 2013 & 12968 & $\mathrm{R} / \mathrm{M}$ & $2000-2005$ & $\begin{array}{l}\text { Evaluate the outcomes in } \\
\text { patients treated by SR, PEI, or } \\
\text { RFA, with no more than } \\
3 \text { tumors and liver } \\
\text { damage of class A or B }\end{array}$ & $\begin{array}{l}\text { OS and time to recurrence } \\
\text { rates were both significantly } \\
\text { better in the SR group than } \\
\text { in the RFA and PEI }\end{array}$ \\
\hline Kim et al. (26) & 2016 & 604 & $\mathrm{R} / \mathrm{S}$ & $2000-2009$ & $\begin{array}{l}\text { Patients with a single } \\
\text { asymptomatic } \mathrm{HCC}<3 \mathrm{~cm} \text { and } \\
\text { good health performance } \\
\text { status, who underwent } \\
\text { liver resection or RFA }\end{array}$ & $\begin{array}{l}\text { RFA treatment was independently } \\
\text { associated with poorer outcomes } \\
\text { in terms of treatment-site } \\
\text { recurrence-free survival }\end{array}$ \\
\hline Xie et al. (27) & 2015 & 70 & $\mathrm{R} / \mathrm{S}$ & $2008-2013$ & $\begin{array}{l}\text { Evaluate the outcomes of } \\
\text { salvage treatments (SS, RFA, } \\
\text { TACE) for RFA-related } \\
\text { local recurrence of HCC }\end{array}$ & $\begin{array}{l}\text { Survival outcomes after } \\
\text { repeated RFA were similar to } \\
\text { those achieved by SS }\end{array}$ \\
\hline Ginsburg et al. (33) & 2015 & 89 & $\mathrm{R} / \mathrm{S}$ & 2003-2011 & $\begin{array}{l}\text { Compare the outcomes and } \\
\text { complications of TACE with } \\
\text { drug-eluting embolic agents } \\
\text { combined with RFA or MW } \\
\text { ablation in treatment of HCC }\end{array}$ & $\begin{array}{l}\text { No statistically significant } \\
\text { difference between those } \\
\text { two approaches in terms of } \\
\text { overall progression-free } \\
\text { survival and OS }\end{array}$ \\
\hline
\end{tabular}

CA: Complete ablation; DR: distant recurrence; HCC: hepatocellular carcinoma; LTP: local tumour progression; M: multicentric; MW: micro wave; OS: overall survival; P: prospective; PEI: percutaneous ethanol injection; R: retrospective; RFA: radiofrequency ablation; RT: randomized trial; S: single center; SR: surgical resection; SS: salvage surgery; TACE: trans-arterial chemo-embolization.

definite evidence of portal hypertension, as assessed clinically. They reported that 5- and 10-year HCC-specific survival rates were $87.6 \%$ and $59.0 \%$ respectively for the resection group with a median survival of 61 months, and $82.1 \%$ and $61.2 \%$ t respectively for the RFA group with a median survival of 66 months $(p=0.214)$. The recurrence rate was higher in the group treated by RFA $(59.8 \%, 198$ patients) than in the group treated by surgical resection $(42.1 \%, 115$ patients) $(p<0.001)$, and the median overall recurrence-free survival was shorter in the RFA group (36 months; $39.4 \%$ at 5 years and $25.1 \%$ at 10 years) than in the resection group (44 months; $60.6 \%$ and $37.5 \%$, respectively) $(p<0.001)$.
The approach to recurrent HCC after RFA was discussed by Xie et al. (27). Among 50 patients that were initially treated by RFA, 27 underwent repeated RFA for local recurrence, 20 underwent salvage surgery, and three underwent TACE. The overall survival rates for patients after RFA were $85.2 \%$ and $52.6 \%$ at 1 and 3 years, respectively, and 91.7 and $78.6 \%$, respectively, for patients after salvage surgery $(p=0.374)$. Data of patients that underwent TACE were unfortunately unavailable. At multivariate analysis, low serum albumin [hazard ratio $(\mathrm{HR})=1.320 ; 95 \%$ confidence interval $(\mathrm{CI})=1.029-1.693 ; p=0.029)$, increased number of tumors $\quad(\mathrm{HR}=1.312 ; 95 \% \quad \mathrm{CI}=1.044-1.649 ; \quad p=0.020)$, 
Table II. Outcomes of the most relevant studies on Trans-Arterial Chemo-Embolization (TACE).

\begin{tabular}{|c|c|c|c|c|c|c|}
\hline Author (Ref) & Year & No. of patients & Type of study & Study interval & Study design & Major outcomes \\
\hline Kirikoshi et al. (34) & 2009 & 253 & $\mathrm{R} / \mathrm{S}$ & $1995-2007$ & $\begin{array}{l}\text { Evaluate the effects of TACE } \\
\text { and of the combined therapies } \\
\text { (TACE + PEI or TACE + RFA) } \\
\text { on the long-term survival rates }\end{array}$ & $\begin{array}{l}\text { TACE alone yielded better } \\
\text { outcomes than palliative } \\
\text { treatment, combined TACE + } \\
\text { PEI/RFA therapy was more } \\
\text { useful than TACE alone for } \\
\text { patients with a single tumor }\end{array}$ \\
\hline Jin et al. (35) & 2014 & 68 & $\mathrm{R} / \mathrm{S}$ & $1998-2012$ & $\begin{array}{l}\text { Compare the treatment } \\
\text { outcomes of surgery/RFA and } \\
\text { TACE in recurrent HCC of } \\
\text { BCLC stage } 0 \text { or A }\end{array}$ & $\begin{array}{l}\text { TACE provides significantly } \\
\text { better OS rate among } \\
\text { recurrent MVI-positive patients } \\
\text { than surgery/RFA after } \\
\text { curative resection for HCC }\end{array}$ \\
\hline Wang et al. (36) & 2015 & 629 & $\mathrm{P} / \mathrm{S}$ & 2004-2010 & $\begin{array}{c}\text { Patients with HCC recurrence } \\
\text { after curative resection were } \\
\text { grouped into re-hepatectomy, } \\
\text { RFA, or TACE }\end{array}$ & $\begin{array}{l}\text { Re-hepatectomy group had } \\
\text { significantly better OS than } \\
\text { the RFA group, and that of the } \\
\text { RFA group was significantly } \\
\text { better than the TACE group }\end{array}$ \\
\hline
\end{tabular}

HCC: Hepatocellular carcinoma; M: multicentric; MVI: microvascular invasion; OS: overall survival; P: prospective; PEI: percutaneous ethanol injection; R: retrospective; RFA: radiofrequency ablation; RT: randomized trial; S: single center; TACE: trans-arterial chemo-embolization.

increased maximum tumor size $(\mathrm{HR}=1.449 ; 95 \% \mathrm{CI}=1.125$ $1.866 ; p=0.004)$, and increased serum AFP level $(\mathrm{HR}=1.336$; $95 \% \mathrm{CI}=1.223-1.527 ; p<0.004)$ were significant prognostic factors for recurrence after initial RFA, while increased number of tumors $(\mathrm{HR}=2.934 ; 95 \% \quad \mathrm{CI}=1.012-8.521$; $p=0.048$ ) was the only significant prognostic factor for recurrence after repeat RFA.

In their work published in 2012, Chang and colleagues discussed the role of RFA after incomplete TACE in a retrospective single-center study of 39 patients (28). The inclusion criteria for the study were: a single HCC smaller than or equal to $5 \mathrm{~cm}$ in diameter; lesions visible on ultrasonography (US), with an acceptable and safe path between the lesion and the skin as observed on the US scan; no extrahepatic metastases present; no imaging evidence of tumor invasion into the major portal or hepatic vein branches; liver cirrhosis classified as CTP class A or B; no history of encephalopathy, ascites refractory to diuretics, or variceal bleeding; and no previous treatment for HCC except TACE. Complete tumor ablation depicted on dynamic CT performed 1 month after treatment was achieved in 36 $(92.3 \%)$ out of 39 patients, with no procedural deaths and a complication rate of $5.1 \%$ (two patients). The overall survival rates at $1,2,3$, and 5 years, were $96.9 \%, 82.9 \%$, $67.8 \%$, and $48.4 \%$, respectively. While increasing tumor diameter was found to be a significant prognostic factor affecting local recurrence $(p=0.047)$, no significant prognostic factors affecting overall survival rate were found.

In 2012, Signoriello et al. reported a multicentric Italian experience comparing the outcomes of patients affected by
HCC and treated with local approaches (29). A total of 425 patients were included, with a median follow-up of 7.7 years. PEI was the most common treatment $(60 \%)$ followed by either TACE or transarterial embolization TAE) (19\%), while RFA was performed in fewer cases. The authors did not find any statistically significant differences in terms of overall survival between the three comparisons they made, namely RFA versus PEI, surgery versus TACE/TAE, and RFA/PEI versus TACE/TAE.

In 2013, Hasegawa and colleagues reported their outcomes from a large cohort of patients who underwent surgical resection, PEI, or RFA, with no more than three tumors and liver damage of class A or B (30). They reported that overall survival rates at 3 and 5 years were 85.3 and $71.1 \%$ in the surgically-resected group, 81.0 and $61.1 \%$ in the RFA group, and 78.9 and $56.3 \%$ in the PEI group; the median survival times were $8.4,5.9$, and 5.6 years in the three groups, respectively. The recurrence rates at 3 and 5 - years in the three groups were 43.3 and 63.8\%, 57.2 and $71.7 \%$, and 64.3 and $76.9 \%$, respectively. These results indicate that the overall survival and recurrence rates were both significantly better in the surgically resected group than in the RFA and PEI groups $(p=0.006$ and $p=0.0001$, $p=0.0001$ and $p=0.0001$, respectively).

Lin and colleagues compared the efficacy of RFA to that of PEI in early-stage HCC in their article published in 2013 (31), in which 1,036 patients from the Taiwan Cancer Registry were included according to the following criteria: $\mathrm{HCC}$ as the primary tumor; stage I or II tumor according to the American Joint Cancer Committee on Cancer (AJCC) 
Table III. Outcomes of the most relevant studies on multimodal treatment.

\begin{tabular}{|c|c|c|c|c|c|c|}
\hline Author (Ref) & Year & n. of patients & Type of study & Study interval & Study design & Major outcomes \\
\hline Shibata et al. (39) & 2009 & 89 & $\mathrm{P}-\mathrm{RT} / \mathrm{S}$ & $2003-2007$ & $\begin{array}{l}\text { Patients with HCC nodules } \\
0.8-3.0 \mathrm{~cm} \text { in diameter were } \\
\text { randomly treated with } \\
\text { combined RFA and TACE } \\
\text { or with RFA alone }\end{array}$ & $\begin{array}{l}\text { No significant difference was } \\
\text { found between the two groups } \\
\text { in terms of local tumor } \\
\text { progression, OS and local } \\
\text { progression-free survival }\end{array}$ \\
\hline Morimoto et al. (40) & 2010 & 37 & $\mathrm{P}-\mathrm{RT} / \mathrm{S}$ & $2005-2009$ & $\begin{array}{l}\text { Patients with solitary HCCs } \\
\text { (diameter, } 3.1-5.0 \mathrm{~cm} \text { ) randomly } \\
\text { assigned to } 2 \text { groups: TACE+ } \\
\text { RFA group, and RFA group }\end{array}$ & $\begin{array}{l}\text { No statistically significant } \\
\text { difference was found between } \\
\text { the two groups in terms of } \\
\text { overall recurrence rate and 1- to } \\
\text { 3-year survival rates ( } p=0.390 \\
\text { and } p=0.369 \text { respectively) }\end{array}$ \\
\hline Kagawa et al. (42) & 2010 & 117 & $\mathrm{R} / \mathrm{S}$ & $2000-2005$ & $\begin{array}{l}\text { Patients receives TACE and } \\
\text { RFA or surgical resection as } \\
\text { the initial curative treatment } \\
\text { for single } \mathrm{HCC}<50 \mathrm{~mm} \text { or } \\
\text { up to } 3 \mathrm{HCCs}<30 \mathrm{~mm}\end{array}$ & $\begin{array}{l}\text { No statistically significant } \\
\text { difference between TACE+RFA } \\
\text { group and surgery group, } \\
\text { while recurrence-free survival } \\
\text { was higher in the surgical } \\
\text { group }(p=0.01)\end{array}$ \\
\hline Kim et al. (41) & 2011 & 123 & $\mathrm{R} / \mathrm{S}$ & 2000-2010 & $\begin{array}{c}\text { Compare the effectiveness } \\
\text { of combined TACE and RFA } \\
\text { with that of RFA alone in patients } \\
\text { with medium sized }(3.1-5.0 \mathrm{~cm}) \\
\text { hepatocellular carcinoma }\end{array}$ & $\begin{array}{l}\text { Cumulative } 1-, 3-, 5-, \text { and } \\
7 \text {-year local tumor progression } \\
\text { rates were significantly lower } \\
\text { in the TACE+RFA than in the } \\
\text { RFA-alone group }(p<0.001)\end{array}$ \\
\hline Kim et al. (43) & 2013 & 84 & $\mathrm{R} / \mathrm{S}$ & $2008-2010$ & $\begin{array}{l}\text { Compare the effectiveness of } \\
\text { RFA combined with TACE } \\
\text { with surgical resection in } \\
\text { patients with a single } \mathrm{HCC} \\
\text { ranging from } 2 \text { to } 5 \mathrm{~cm}\end{array}$ & $\begin{array}{l}\text { RFA combined with TACE gave } \\
\text { similar results in terms of } \\
\text { recurrence-free and OS rates }\end{array}$ \\
\hline Yin et al. (44) & 2015 & 211 & $\mathrm{R} / \mathrm{S}$ & $2005-2011$ & $\begin{array}{l}\text { Evaluate the effectiveness } \\
\text { of RFA in patients with } \\
\text { intermediate (BCLC B) stage } \\
\text { hepatocellular carcinoma who } \\
\text { underwent TACE }\end{array}$ & $\begin{array}{l}\text { Post treatment } 1-, 3-\text { and } \\
5 \text {-year cumulative OS was } \\
89.8 \%, 61.1 \% \text { and } 37.4 \% \\
\text { respectively in TACE+RFA } \\
\text { group versus } 67.2 \%, 36.6 \% \\
\text { and } 16.5 \% \text { in TACE } \\
\text { alone group }(p=0.01)\end{array}$ \\
\hline
\end{tabular}

HCC: Hepatocellular carcinoma; M: multicentric; MVI: microvascular invasion; OS: overall survival; P: prospective; PEI: percutaneous ethanol injection; R: retrospective; RFA: radiofrequency ablation; RT: randomized trial; S: single center; TACE: trans-arterial chemo-embolization.

system, 6th edition; PEI or RFA as the first course of treatment within one year of diagnosis; age $>18$ years. The exclusion criteria were as follow: reported prior cancer; multiple primary cancers; histological type with lymphoma (M-code: 9590-9989), or Kaposi's sarcoma (M-code: 9140). RFA led to a significantly better overall survival than PEI, $83 \%$ and $71 \%$, respectively, at 2 years post-diagnosis and $55 \%$ and $42 \%$ at 5 years $(p<0.001)$.

Zhang and colleagues reported the outcomes of 155 patients who underwent either RFA (78 patients) or microwave ablation (77 patients) in a retrospective study (32). Inclusion criteria were a solitary $\mathrm{HCC}$ lesion of $5 \mathrm{~cm}$ in greatest diameter or smaller; three or fewer multiple HCC lesions with a greatest diameter of $3 \mathrm{~cm}$ or less; no radiological evidence of vascular invasion and extrahepatic metastases; index tumors with no prior treatment; liver function classed as CTP class A; prothrombin time of less than $22 \mathrm{~s}$ and platelet count higher than 45 cells6109/1; no history of ascites refractory to diuretics, variceal bleeding, or encephalopathy. The reported 1-, 3-, and 5-year overall survival rates were $91.0 \%, 64.1 \%$ and $41.3 \%$, respectively, for the RFA group and $92.2 \%, 51.7 \%$, and $38.5 \%$ for the microwave ablation group, without any statistically significant difference $(p=0.780)$. The $1-, 3$-, and 5-year disease-free survival rates were $70.5 \%, 42.3 \%$, and $34.2 \%$, respectively, for the RFA group and $62.3 \%, 33.8 \%$, and $20.8 \%$ for the microwave ablation group; even in this case no statistically significant difference was noted. Interestingly, for the subgroup of patients with tumor diameters of $3.1-5.0 \mathrm{~cm}$, the disease-free survival curve for the RFA-treated group was 
significantly better than that for the microwave ablationtreated group (log-rank test, $p=0.018$ ).

Later in 2015, Ginsburg and colleagues compared the efficacy of a combined TACE plus RFA therapy versus TACE plus microwave ablation (33). Eighty-nine patients with HCC received TACE plus RFA (38 patients) or TACE plus microwave ablation (51 patients), without any statistically significant difference between the two groups in patient demographics, including age, sex, and etiology of liver cirrhosis, and no significant difference in tumor characteristics, including tumor size, number of tumors, and BCLC staging. The authors did not find any statistically significant difference between these two approaches in terms of overall progression-free survival and overall survival.

TACE. Kirikoshi and colleagues reported a retrospective study in 2009 which compared the effects of TACE and of combined therapies (TACE plus PEI or TACE plus RFA) on long-term survival (34). The following eligibility criteria were employed:previously untreated patients, and no history of previous surgical treatments such as hepatic resection or liver transplantation. Exclusion criteria were as follow: poor hepatic function (CTP class C, especially presence of poorly controlled hepatic encephalopathy or ascites); presence of extra-hepatic metastasis; vascular contraindications to chemoembolization (hepatic artery thrombosis, main portal vein thrombosis, arteriovenous shunting); poor performance status. Patients with multiple nodules $(<3)$ having a diameter $<3 \mathrm{~cm}$ each were treated by PEI or RFA alone when all the lesions could be identified using ultrasound. If not, or in patients with more than three nodules, TACE alone was performed. Combined therapy (TACE plus PEI/RFA) was administered to the following patients: those with nodules fulfilling the Milan Criteria, but in whom some of the HCC lesions could not be detected by ultrasound; those with a single lesion measuring more than $3 \mathrm{~cm}$ in diameter; those with a large main lesion with small intra-hepatic metastasis and more than three lesions. When TACE alone was compared to palliative group, the median survival time was significantly superior [24.9 months (range $=2-30$ months), $v s$. 10.4 months (range $=2-63$ months), $p=0.0006]$. Moreover, the cumulative survival rates in the TACE-alone group were significantly superior to those in the palliative treatment group, being $98.2 \%$ at 6 months, $90.2 \%$ at 1 year, $55.9 \%$ at 2 years and $16.3 \%$ at 5 years $(p<0.0001)$. When comparing combined therapy to TACE alone, both the median survival time [46.6 months (range=9-114 months) vs. 24.9 months (range 2-30 months), $p<0.0001]$ and the cumulative survival rates $(100 \%, 97.2 \%, 86.7 \%$ and $53.5 \%$ versus $98.2 \%, 90.2 \%$, $55.9 \%$ and $16.3 \%$ at 6-months, and 1,2 and 5 years, respectively, $p<0.0001)$ were in favor of the combinedtherapy group. On the other hand, the authors did not find any statistically significant difference when comparing RFA/PEI to combined therapy ( $p=0.09$ and $p=0.72$ for median survival time and cumulative survival rates, respectively).

In 2014, Jin and colleagues in their retrospective study reported the outcomes of 68 patients who underwent either TACE or surgery/RFA for recurrent HCC after an initial surgical approach (35). Cumulative overall survival rates at 1,3 , and 5 years after re-treatment were $65.1 \%, 27.9 \%$ and $18.6 \%$, respectively, in the surgery/RFA group, and $91.2 \%$, $66.1 \%$ and $30.0 \%$, respectively, in the TACE group $(p=0.23)$. They also performed a subgroup analysis of patients treated with no residual disease after treatment (so-called 'R0' resection): although with a marginal statistical significance, patients with microvascular infiltration (MVI) treated with TACE (10 patients) had a higher recurrence-free survival than patients who underwent surgery/RFA $(p=0.054)$. Overall survival in patients with MVI was also significantly higher in the TACE-treated group versus that treated with surgery/RFA $(p=0.01)$. Wang and colleagues published a prospective study in 2015 comparing surgery, RFA and TACE in patients who developed intrahepatic tumor recurrence after partial hepatectomy (36). They used the following inclusion criteria: R0 resection for primary HCC; recurrent tumors met the Milan criteria; no evidence of extrahepatic metastasis; liver function of CTP class score $<8$, without severe esophageal varices; re-hepatectomy, RFA or TACE as the first-line treatment for recurrence. The results show that the 1,3, and 5-year overall survival rates were $97.7 \%, 84.1 \%$ and $64.5 \%$ for the re-hepatectomy group, $88.4 \%, 55.8 \%$ and $27.7 \%$ for the TACE group, and $96.9 \%$, $73.4 \%$ and $37.0 \%$ for the RFA group (re-hepatectomy $v$. TACE, $p<0.001 ; v s$. RFA, $p=0.005$; TACE $v s$. RFA, $p<0.001)$. The 1,3-, and 5-year recurrence to death survival (RTDS) rates were $94.5 \%, 71.5 \%$ and $43.0 \%$ for the rehepatectomy group, $76.6 \%, 39.3 \%$ and $8.3 \%$ for the TACE group, and $90.4 \%, 53.7 \%$ and $26.7 \%$ for the RFA group (rehepatectomy vs. TACE, $p<0.001$; vs. RFA, $p=0.008$; TACE $v s$. RFA, $p<0.001)$.

Radioembolization. Khor et al. in 2014 reported a retrospective study of patients, all with normal renal function, with unresectable tumors and portal vein thrombosis (PVT) or tumors larger than $3 \mathrm{~cm}$ that were not amenable to RFA who were considered for radioembolization (37). The median reported overall survival was 14.4 months (95\% CI=11.0-22.2 months). They clarified that patients with CTP class A disease fared better compared to those with CTP class B disease (21.7 vs. 7.1 months, respectively; $p=0.0001)$. Moreover, patients without vascular invasion had significantly better median survival compared to patients with vascular invasion (18.1 vs. 11.0 months, respectively; $p=0.02$ ).

Bhangoo et al. in 2015 reported the effects of yttrium-90 (Y-90) radioembolization in a population of 17 patients with unresectable HCC who either had failed or had disease not amenable to alternative locoregional therapies (38). The 
inclusion criteria were age of 18 years or more; ECOG performance status 0-2; serum total bilirubin less than $2 \mathrm{mg} / \mathrm{l}$; ability to undergo angiography. Conversely, exclusion criteria included were uncorrectable flow to the gastrointestinal tract, significant extrahepatic disease, applied lung dose greater than 30 Gy in a single fraction, and CTP class C cirrhosis. The authors reported a median survival of 8.4 months (range $=1.3$ to 21.1 months) after their first radioembolization treatment, and a mean overall survival from the time of diagnosis of 11.7 months (range=3.4-43.2 months).

Multimodal treatments. In 2009, Shibata and colleagues reported their experience in a prospective randomized trial comparing TACE plus RFA versus RFA alone in patients fulfilling the following inclusion criteria: three or fewer HCC nodules $3 \mathrm{~cm}$ in diameter or smaller, CTP class A or B cirrhosis, no imaging evidence of tumor invasion into the major portal or hepatic vein branches, no extrahepatic metastasis, and a platelet count of more than $40,000 \mathrm{cells} / \mathrm{mm}^{3}$ (39). A total of 89 patients entered the study, 46 in the combined therapy group and 43 in that for RFA alone. Local tumor progression, overall survival and local progression-free survival rates at 1-, 2-, 3and 4-years did not differ significantly between the RFA group and combined-treatment group ( $p=0.797, p=0.515$ and $p=0.934$, respectively).

Morimoto and colleagues presented a randomized trial in 2010 also comparing the midterm outcomes of TACE plus RFA versus RFA alone (40). The following inclusion criteria were met by 37 patients that did not receive any other previous treatment for HCC: ECOG performance status score of 2 or less; CTP class A or B; solitary lesion; maximum tumor diameter of $3.1-5.0 \mathrm{~cm}$; lesion detected by ultrasonography; divergence of the hepatic artery suitable for TACE; no evidence of portal or venous thrombosis, extrahepatic metastasis, or uncontrollable ascites; adequate hematologic function (platelet count $>50 \times 10^{9}$ cells $/$, hemoglobin $>8.0 \mathrm{~g} / \mathrm{dl}$, and prothrombin time $<80 \%$ ); adequate hepatic function (albumin $>2.5 \mathrm{~g} / \mathrm{dl}$, total bilirubin $<3 \mathrm{mg} / \mathrm{dl}$, and alanine aminotransferase and aspartate aminotransferase levels $<5 \times$ the upper limit of the normal range); and adequate renal function (serum creatinine concentration $<1.5 \times$ the upper limit of the normal range). A total of 18 patients were assigned to the RFA group, and 19 to the TACE-RFA group, technical success was obtained in all 37 patients. Local tumor progression rate in the RFA group was 39\% at the end of the first, second, and third years, while in the TACE-RFA group it was correspondingly 6\% (log-rank test, $p=0.012$ ). Conversely, no statistically significant difference was found between the two groups in terms of overall recurrence rate and 1-to 3-year survival rates ( $p=0.390$ and $p=0.369$ respectively).

Later in 2011, Kim and colleagues reported their experience with a cohort of 123 patients to compare the effectiveness of combined TACE and RFA with that of RFA alone in patients with medium-sized $(3.1-5.0 \mathrm{~cm})$ hepatocellular carcinoma (41). Patients were eligible to be included in the study if they had a single HCC $>3 \mathrm{~cm}$ but $\leq 5 \mathrm{~cm}$ in the largest diameter, no imaging evidence of vascular invasion by the tumor, and no evidence of extrahepatic disease. Patients also were included if they were not candidates for surgical resection based on the liver function, if they had unpredictable transplantation time during waiting period for liver transplantation, or if they refused to undergo surgery or liver transplantation. From March 2000 to March 2010, 66 patients underwent RFA alone and 57 patients TACE plus RFA. The cumulative 1-, 3-, 5-, and 7-year local tumor progression rates in the TACE plus RFA group were $9 \%, 40 \%, 55 \%$ and $66 \%$, respectively, significantly lower than the rates of $45 \%, 76 \%, 86 \%$, and $89 \%$, respectively, in the RFA-alone group $(p<0.001)$. At multivariate analysis, treatment allocation $(\mathrm{OR}=1.78 ; 95 \%$ $\mathrm{CI}=1.12-2.83 ; p=0.016)$ and CTP class $(\mathrm{OR}=1.96 ; 95 \%$ $\mathrm{CI}=1.19-3.23 ; p=0.008)$ were significant independent factors associated with patient survival.

In 2010, Kagawa and colleagues reported the outcomes of a study involving 117 patients undergoing TACE followed by RFA within 2 months (62 patients) or surgery alone (55 patients) for early HCC (42). Inclusion criteria were no previous treatment for $\mathrm{HCC}$, a single $\mathrm{HCC}<50 \mathrm{~mm}$ or up to three HCCs $<30 \mathrm{~mm}$, cirrhosis classified as CTP class A, no vascular invasion, and no extrahepatic metastasis, while exclusion criteria were ECOG performance status $>2$ and presence of an uncontrollable malignancy other than HCC. Overall survival at 1,3 , and 5 years were $100 \%, 94.8 \%$ and $64.6 \%$ in the TACE plus RFA group, and $92.5 \%, 82.7 \%$ and $76.9 \%$ in the resection group, respectively, without any statistically significant difference ( $p=0.788$ by log-rank test). Conversely, recurrence-free survival rates at 1,3 , and 5 years were $64.5 \%, 40.1 \%$ and $18.0 \%$ in the TACE-RFA group, and $75.6 \%, 41.1 \%$, and $36.4 \%$ in the resection group, respectively ( $p=0.01$ by log-rank test).

Similarly in 2013, Kim et al. compared the outcomes of TACE combined with RFA versus surgery alone in a series of 84 patients affected by HCC (43). The following inclusion criteria were considered for that study: a single HCC of $2-5 \mathrm{~cm}$ in the largest dimension, no history of prior treatment for HCC, no major vascular invasion and no evidence of extrahepatic tumor. The length of hospital stay after the procedure was significantly longer in the surgically resected group (19.8 8 8.4 days) than in the combined therapy group $(7.4 \pm 2.2$ days $)(p<0.0001)$. Recurrence-free survival rates at $1,2,3$ and 4 years were $89.2 \%, 75.2 \%, 69.4 \%$ and $69.4 \%$, respectively, in the combined-therapy group and $81.8 \%$, $68.5 \%, 68.5 \%$ and $65 \%$, respectively, in the surgicallyresected group, without any statistically significant difference between the two groups $(p=0.7962)$. Overall survival rates at $1,2,3$ and 4 years were not significantly 
different $(p=0.63210)$, as they were $97.3 \%, 86.5 \%, 78.4 \%$ and $78.4 \%$, respectively, in the combined therapy group, and $95.7 \%, 89.4 \%, 84.3 \%$ and $80.3 \%$, respectively, in the surgical resection group.

In their article, Yin and colleagues present a single-center study of patients undergoing TACE alone versus TACE and RFA (44). They reported the following inclusion criteria: the presence of a single $\mathrm{HCC}$ tumor $\leq 8 \mathrm{~cm}$ in diameter, or multinodular HCC tumors $(\mathrm{n} \leq 5)$ smaller than $5 \mathrm{~cm}$ in diameter before initial TACE; the presence of viable residual HCC with retained iodized oil after TACE as shown by the followup liver CT and/or MRI scan; the absence of portal vein invasion and extrahepatic metastasis; and CTP class A or B. 55 patients received combined RFA treatment based on the following criteria: viable residual tumors after TACE could be detected by follow-up ultrasonography, residual tumors could be possibly ablated with curative intention by RFA; absence of severe coagulopathies, such as prothrombin time $\leq 16$ s or platelet count $>50,000 / \mathrm{ml}$; and patients who signed informed consent for RFA. The other 156 patients who were not suitable for RFA and received repeated TACE treatment were assigned to TACE alone group based on the following reasons: tumors were poorly visible on planning ultrasound; percutaneous RFA was infeasible due to the high-risk location of thermal injury or could result in incomplete ablation due to the inadequate electrode path; coagulopathy such as prothrombin time $>16$ s or platelet count $<50,000 / \mathrm{ml}$; and unwillingness to receive additional RFA treatment due to economic or other personal reasons, although residual tumors could be treated with combined RFA. The results showed that in TACE plus RFA group $(n=55)$, complete response, partial response, stable disease and progressive disease rates were $60 \%, 10.9 \%$ and $3.6 \%$ and $25.5 \%$, respectively, versus $11.5 \%, 20.5 \%, 22.4 \%$ and $45.5 \%$, respectively, in TACE alone group $(n=156)$. Moreover, the reported cumulative 1-, 3-, and 5-year tumor progression rates in TACE alone group were $71.4 \%, 98.3 \%$ and $100 \%$, respectively, which were significantly higher than $36.0 \%$, $81.6 \%$ and $90.8 \%$ in the TACE+RFA group $(p<0.001)$. Lastly, the post treatment 1-, 3- and 5-year cumulative overall survival rates were $89.8 \%, 61.1 \%$ and $37.4 \%$, respectively, in TACE+RFA group versus $67.2 \%, 36.6 \%$ and $16.5 \%$ in TACE alone group $(p=0.01)$.

\section{Discussion}

Surgical resection remains the standard treatment for patients with a single nodule, preserved liver function, and good performance status. According to the most recent data, it is associated with 5-year survival rates up to $70 \%$ and $2 \%$ to $3 \%$ perioperative mortality in patients with cirrhosis. Moreover, some authors report 5-year survival rates above $50 \%$ in patients undergoing resection for a single lesion $\leq 5 \mathrm{~cm}$, or up to three lesions $\leq 3 \mathrm{~cm}$ each [Milan criteria (45)] but who were not fit for transplantation (46). As previously reported, laparoscopic technique with an accurate ultrasound examination of liver parenchyma can allow for a complete exclusion of hepatic lesions undetectable at the preoperative imaging and provides the minimal onset of adhesions, which is extremely useful in patients undergoing liver transplantation (47). However, liver transplantation performed in patients fulfilling the Milan criteria results in a 5-year overall survival rate of $75 \%$, with a risk of recurrence of $<15 \%$, with perioperative mortality and 1-year mortality of approximately $3 \%$ and $\leq 10 \%$, respectively (46). As a matter of fact, liver transplantation is the most effective therapy for cirrhosis, the underlying liver disease that causes HCC (48). Therefore what is the role of local treatment of HCC?

As reported by Wang et al. in a recent review, PEI and RFA are widely used in clinical practice (49). The major pitfalls of these two techniques are the poor efficacy of PEI in tumors greater than $2 \mathrm{~cm}$ and the use of RFA in tumors close to major vessels. This is due to the distribution of ethanol that may be blocked by the intratumoral fibrotic septa or the tumor capsule, resulting in a heterogeneous distribution. Conversely, RFA results in coagulative necrosis of both the tumor and the surrounding parenchymal tissue, also being effective on small undetected satellites. Besides the potential vascular damage when tumors are close to great vessels, the heat-sink effect may result in incomplete ablation (16). However, RFA has been shown to be as effective as hepatic resection in the treatment of small singlenodule HCC. Moreover, it has been shown that RFA is related to better overall survival compared to PEI regardless of tumor size (31).

Even if not statistically significant, data reported by Kim et al. (26) show that surgery cannot be replaced by RFA alone, especially in patients without severe liver disease who are therefore amenable to radical treatments without high risk of morbidity. The role of RFA for recurrent HCC after front-line RFA has also been studied. We herein reported data from Xie and colleagues that showed how RFA is comparable to salvage liver resection (27). One major limitation of their study is the bias in the patient selection process: panel discussion with multidisciplinary treatment team including surgeons, radiologists, oncologists, and pathologists was performed to make a decision regarding the optimal treatment modality for local recurrence. According to the authors, RFA was recommended as the first choice for favorable local recurrence (defined as tumor size $\leq 5 \mathrm{~cm}$, focality around prior ablation site, absence of macrovascular invasion, and located $>5 \mathrm{~mm}$ from important structures); TACE was an alternative treatment for patients with concurrent multiple or large intrahepatic metastases; when local non-surgical treatment was deemed infeasible or failed, salvage liver resection was recommended when liver 
function reserve was enough and all radiology-found tumors were located within one lobe. Similarly, according to Chang and colleagues, RFA can improve long-term outcomes after incomplete TACE, with results comparable to RFA alone (28). No statistically significant difference was demonstrated in terms of overall survival when comparing RFA versus $\mathrm{PEI}$, surgery versus TACE/TAE, and RFA/PEI versus TACE/TAE according Signoriello and colleagues (29). This study, an interesting attempt to collect multicentric data from an Italian region, has a major limitation in being retrospective and grouping together all HCCs regardless of size, without specifying the indication for each treatment. Therefore, such data may be confusing.

TACE is reported to be more effective than surgery or RFA in patients with early recurrence and MVI after curative resection, staged as BCLC 0 or A (35). Despite its retrospective setting, lack of randomization and small sample size, this article contributed to the discussion on which treatment should be preferred for recurrent HCCs as a bridge to salvage transplantation. As previously reported, the absence of active residual HCC in the native liver at histological examination after liver transplantation in patients who had undergone bridging therapies was a highly positive prognostic factor in regard to $\mathrm{HCC}$ recurrence and overall patient survival (50). Moreover, an aggressive treatment of HCC with liver resection, TACE, and RFA is necessary for down-staging and is able to qualify patients for transplant (51). Later in 2015, in a prospective study Wang and colleagues reported that in patients treated for recurrence after curative hemi-hepatectomy, the repeat hepatectomy group had significantly better overall survival and RTDS than the RFA group, and that of the RFA group was significantly better than that of the TACE group (36).

The approach with TACE versus palliative care in patients with more than three HCC nodules showed significantly greater efficacy according to Kirikoshi and colleagues (34). However, combined therapy with TACE plus PEI/RFA resulted in better outcomes versus TACE alone, also when exceeding the Milan Criteria $(p=0.0024)$. The authors also reported that there was no statistically significant difference between PEI/RFA alone versus combined therapy. Although the setting of this study was not prospective, with potential biases due to its retrospective nature, it shows that combined therapies are not superior to RFA alone for intermediate HCCs. More data about this interesting topic came from other articles presenting multimodal approaches to resectable HCC. In $2011 \mathrm{Kim}$ and colleagues reported that cumulative 1-, 3-, 5-, and 7-year local tumor progression rates were significantly lower in the TACE+RFA than in the RFA-alone group $(p<0.001)$ in patients with medium-sized HCC (41). Although remarkable, the results of this study are biased by its retrospective and non-randomized design. Kagawa and Kim reported their results comparing surgical resection and combined approach of TACE followed by RFA. They reported no differences in terms of overall survival, while recurrencefree survival was in favor of the surgical approach $(p=0.01)$, whereas in the study from Kim and colleagues, no significant difference was found in terms of recurrence-free and overall survival between the two approaches $(42,43)$. The two studies differ for the timing of RFA, which was scheduled on the day after TACE in the study by Kim et al., while in the study of Kagawa et al., it was performed within 2 months of TACE. In both cases, the results are biased from the small number of patients and the non-randomized nature of the studies. According to Kim et al., indication to perform either surgical or local treatment was given by an institution hepatobiliary team, excluding from surgery patients that wanted to undergo nonsurgical treatment $(n=22)$, patients with inappropriate hepatic functional reserve $(n=9)$, and patients with small volume of remnant liver after surgery $(n=6)(43)$. In their well-written article, Yin and colleagues reported that the total tumor control rate was $74.5 \%$ in the TACE plus RFA group versus $54.5 \%$ in the TACE alone group $(p<0.001)(44)$. After stratification for tumor number and size, neither 1-, 3-, and 5year tumor progression rates nor overall survival significantly differed between the single-tumor group and multi-tumor group. It seems relevant that the two randomized trials reported $(39,40)$ failed to demonstrate a statistically significant advantage for the TACE plus RFA combination versus RFA alone in a cohort of small and intermediate-sized HCCs. As a matter of fact, Morimoto and colleagues demonstrated that such a combination therapy is safe and effective for extending the ablated area, thereby contributing to restricting local tumor progression, but a larger number of patients may be needed to confirm the survival benefits (40). Conversely, Shibata et al. showed that the addition of TACE to RFA for HCCs of $3 \mathrm{~cm}$ or smaller does not appear to be warranted since local tumor progression rates, overall survival rates, and local progression-free survival rates were equivalent to those of patients who underwent RFA alone (39).

As previously underlined, further studies should evaluate the applicability, the safety and the outcome after aggressive treatments in patients with advanced HCC, such as portal vein embolization, prior extended hepatectomies, combined locoregional treatments and chemotherapy protocols in our constantly aging society (52). For example, sarcopenia was associated with worse outcomes in patients who underwent liver resection or liver transplantation for $\mathrm{HCC}$ and intrahepatic cholangiocarcinoma, with an incidence of postoperative complications of $40.4 \%$ among patients with sarcopenia versus $18.4 \%$ among patients without $(p=0.01)$ (53). In an interesting article published in 2013, Nathan and colleagues addressed the hypothesis that non-clinical factors, i.e. hospital- or surgeonrelated factors, may drive the decision of which approach should be adopted for early HCCs (54). Four major groups for analysis were considered: liver transplantation and RFA, with 
or without liver resection (41\%); liver transplantation but not RFA, with or without liver resection (14\%); liver resection and RFA, but not liver transplantation (39\%); and liver resection but not RFA or liver transplantation (6\%). Significant predictors of choice of therapy included practice type, with academic surgeons favoring liver resection (relative risk ratio $=1.71 ; 95 \% \mathrm{CI}=0.97-3.02$ ) and $\mathrm{LT}$ (relative risk ratio=2.42; 95\% $\mathrm{CI}=1.34-4.36)$ over RFA more strongly than privatepractice surgeons $(p=0.09)$. Annual HCC patient volume also impacted choice of therapy, with higher-volume surgeons favoring transplantation over resection. Of note, nontransplantation surgeons were more likely than transplantation surgeons to choose resection and RFA over transplantation. However, surgeons who did not personally perform transplantation but worked at hospitals that offered it were less likely to choose RFA than those who worked at hospitals without transplantation $(p=0.001)$. This clearly reflects the confusion present in literature and the lack of definitive data.

\section{Conclusion}

Data in the literature are confusing and difficult to compare due to the lack of prospective studies. Overall, we believe that a multidisciplinary panel should provide a tailored approach for each patient, considering both guideline indications and patient-specific characteristics, and enhancing the hospital-specific best practice. The trend in the evaluation of HCC is still bi-dimensional, focusing on tumor dimension and number as prognostic factors and key elements in the decision-making process of HCC. We believe that in the near future' the genetic fingerprint of HCC will play a growing role, modifying both staging and indications for treatment, according to a new three-dimensional system.

\section{References}

1 Tejeda-Maldonado J, García-Juárez I, Aguirre-Valadez J, González-Aguirre A, Vilatobá-Chapa M, Armengol-Alonso A, Escobar-Penagos F, Torre A, Sánchez-Ávila JF and CarrilloPérez DL: Diagnosis and treatment of hepatocellular carcinoma: An update. World J Hepatol 7(3): 362-376, 2015.

2 El-Serag HB and Kanwal F: Epidemiology of hepatocellular carcinoma in the United States: where are we? Where do we go? Hepatology 60(5): 1767-1775, 2014.

3 Santi V, Buccione D, Di Micoli A, Fatti G, Frigerio M, Farinati F, Del Poggio P, Rapaccini G, Di Nolfo MA, Benvegnù L, Zoli M, Borzio F, Giannini EG, Caturelli E, Chiaramonte M, Bernardi $\mathrm{M}$ and Trevisani $\mathrm{F}$ : The changing scenario of hepatocellular carcinoma over the last two decades in Italy. J Hepatol 56(2): 397-405, 2012.

4 Borzio M, Dionigi E, Rossini A, Toldi A, Francica G, Fornari F, Salmi A, Farinati F, Vicari S, Marignani M, Terracciano F, Ginanni B and Sacco R: Trend of improving prognosis of hepatocellular carcinoma in clinical practice: an Italian in-field experience. Dig Dis Sci 60(5): 1465-1473, 2015.
5 Blonski W, Kotlyar DS and Forde KA: Non-viral causes of hepatocellular carcinoma. World J Gastroenterol 16(29): 36033615, 2010.

6 Sherman M: Modern approach to hepatocellular carcinoma. Curr Gastroenterol Rep 13(1): 49-55, 2011.

7 Di Francia R, Rinaldi L, Troisi A, Di Benedetto F and Berretta M: Effect of anti-oxidant agents in patients with hepatocellular diseases. Eur Rev Med Pharmacol Sci 19(21): 3993-3995, 2015.

8 Berretta M, Garlassi E, Cacopardo B, Cappellani A, Guaraldi G, Cocchi S, De Paoli P, Lleshi A, Izzi I, Torresin A, Di Gangi P, Pietrangelo A, Ferrari M, Bearz A, Berretta S, Nasti G, Di Benedetto F, Balestreri L, Tirelli $U$ and Ventura $P$ : Hepatocellular carcinoma in HIV-infected patients: check early, treat hard. Oncologist 16(9): 1258-1269, 2011.

9 Chiba T, Suzuki E, Saito T, Ogasawara S, Ooka Y, Tawada A, Iwama $\mathrm{A}$ and Yokosuka O: Biological features and biomarkers in hepatocellular carcinoma. World J Hepatol 7(16): 2020-2028, 2015.

10 Zucman-Rossi J, Villanueva A, Nault JC and Llovet JM: Genetic Landscape and Biomarkers of Hepatocellular Carcinoma. Gastroenterology 149(5): 1226-1239 e4, 2015.

11 Magistri P, Leonard SY, Tang CM, Chan JC, Lee TE and Sicklick JK: The glypican 3 hepatocellular carcinoma marker regulates human hepatic stellate cells via Hedgehog signaling. J Surg Res 187(2): 377-385, 2014.

12 Sicklick JK: Correcting the misnomers of epithelialmesenchymal relations. J Surg Res 182(1): 36-39, 2013.

13 Sicklick JK, Li YX, Choi SS, Qi Y, Chen W, Bustamante M, Huang J, Zdanowicz M, Camp T, Torbenson MS, Rojkind M and Diehl AM: Role for hedgehog signaling in hepatic stellate cell activation and viability. Lab Invest 85(11): 1368-1380, 2005.

14 Guerrieri F: Long non-coding RNAs era in liver cancer. World J Hepatol 7(16): 1971-1973, 2015.

15 Page AJ, Cosgrove DC, Philosophe B and Pawlik TM: Hepatocellular carcinoma: diagnosis, management, and prognosis. Surg Oncol Clin N Am 23(2): 289-311, 2014.

16 de Lope CR, Tremosini S, Forner A, Reig M and Bruix J: Management of HCC. J Hepatol 56(Suppl 1): S75-87, 2012.

17 Reichman TW, Bahramipour P, Barone A, Koneru B, Fisher A, Contractor D, Wilson D, Dela Torre A, Cho KC, Samanta A and Harrison LE: Hepatitis status, child-pugh classification, and serum AFP levels predict survival in patients treated with transarterial embolization for unresectable hepatocellular carcinoma. J Gastrointest Surg 9(5): 638-645, 2005.

18 Huo TI, Lin HC, Hsia CY, Wu JC, Lee PC, Chi CW and Lee SD: The model for end-stage liver disease based cancer staging systems are better prognostic models for hepatocellular carcinoma: a prospective sequential survey. Am J Gastroenterol 102(9): 1920-1930, 2007.

19 Kee KM, Wang JH, Lin CY, Wang CC, Cheng YF and Lu SN: Validation of the 7 th edition TNM staging system for hepatocellular carcinoma: an analysis of 8,828 patients in a single medical center. Dig Dis Sci 58(9): 2721-2728, 2013.

20 Pawlik TM, Delman KA, Vauthey JN, Nagorney DM, Ng IO, Ikai I, Yamaoka Y, Belghiti J, Lauwers GY, Poon RT and Abdalla EK: Tumor size predicts vascular invasion and histologic grade: Implications for selection of surgical treatment for hepatocellular carcinoma. Liver Transpl 11(9): 1086-1092, 2005. 
21 Hsu CY, Lee YH, Hsia CY, Huang YH, Su CW, Lin HC, Lee RC, Chiou YY, Lee FY and Huo TI: Performance status in patients with hepatocellular carcinoma: determinants, prognostic impact, and ability to improve the Barcelona Clinic Liver Cancer system. Hepatology 57(1): 112-119, 2013.

22 Llovet JM, Bru C, and Bruix J: Prognosis of hepatocellular carcinoma: the BCLC staging classification. Semin Liver Dis 19(3): 329-338, 1999.

23 Di Benedetto F, Di Sandro S, D’Amico G, De Santis M and Gerunda GE: Role of chemoembolization as a rescue treatment for recurrence of resected hepatoblastoma in adult patients. Surg Innov 18(2): 136-140, 2011

24 Berretta M, Di Francia R, and Tirelli U: Sorafenib combined with percutaneous radiofrequency ablation for the treatment of medium-sized hepatocellular carcinoma. Eur Rev Med Pharmacol Sci 19(14): 2521-2522, 2015.

25 Moher D, Liberati A, Tetzlaff J, Altman DG and The PRISMA Group: Preferred Reporting Items for Systematic Reviews and Meta-Analyses: The PRISMA Statement. BMJ 339: b2535, 2009.

26 Kim GA, Shim JH, Kim MJ, Kim SY, Won HJ, Shin YM, Kim PN, Kim KH, Lee SG and Lee HC: Radiofrequency ablation as an alternative to hepatic resection for single small hepatocellular carcinomas. Br J Surg 103(1): 126-135, 2016

27 Xie X, Jiang C, Peng Z, Liu B, Hu W, Wang Y, Lin M, Lu M and Kuang M: Local Recurrence after Radiofrequency Ablation of Hepatocellular Carcinoma: Treatment Choice and Outcome. J Gastrointest Surg 19(8): 1466-1475, 2015.

28 Chang NK, Shin SS, Kim JW, Kim HJ, Jeong YY, Heo SH, Kim JK and Kang HK: Effect of ultrasound-guided radiofrequency ablation in incompletely treated hepatocellular carcinoma after transcatheter arterial chemoembolization. Korean J Radiol 13(Suppl 1): S104-111, 2012.

29 Signoriello S, Annunziata A, Lama N, Signoriello G, Chiodini P, De Sio I, Daniele B, Di Costanzo GG, Calise F, Olivieri G, Castaldo V, Lanzetta R, Piai G, Marone G, Visconti M, Fusco M, Di Maio M, Perrone F, Gallo C and Gaeta GB: Survival after locoregional treatments for hepatocellular carcinoma: a cohort study in real-world patients. Scientific World J 2012: 564706, 2012 .

30 Hasegawa K, Kokudo N, Makuuchi M, Izumi N, Ichida T, Kudo M, Ku Y, Sakamoto M, Nakashima O, Matsui O and Matsuyama Y: Comparison of resection and ablation for hepatocellular carcinoma: a cohort study based on a Japanese nationwide survey. J Hepatol 58(4): 724-729, 2013.

31 Lin ZZ, Shau WY, Hsu C, Shao YY, Yeh YC, Kuo RN, Hsu CH, Yang JC, Cheng AL and Lai MS: Radiofrequency ablation is superior to ethanol injection in early-stage hepatocellular carcinoma irrespective of tumor size. PLoS One 8(11): e80276, 2013.

32 Zhang L, Wang N, Shen Q, Cheng W and Qian GJ: Therapeutic efficacy of percutaneous radiofrequency ablation versus microwave ablation for hepatocellular carcinoma. PLoS One 8(10): e76119, 2013.

33 Ginsburg M, Zivin SP, Wroblewski K, Doshi T, Vasnani RJ and Van Ha TG: Comparison of combination therapies in the management of hepatocellular carcinoma: transarterial chemoembolization with radiofrequency ablation versus microwave ablation. J Vasc Interv Radiol 26(3): 330-341, 2015.
34 Kirikoshi H, Saito S, Yoneda M, Fujita K, Mawatari H, Uchiyama T, Higurashi T, Goto A, Takahashi H, Abe Y, Inamori M, Kobayashi N, Kubota K, Sakaguchi T, Ueno N and Nakajima A: Outcome of transarterial chemoembolization monotherapy, and in combination with percutaneous ethanol injection, or radiofrequency ablation therapy for hepatocellular carcinoma. Hepatol Res 39(6): 553-562, 2009.

35 Jin YJ, Lee JW, Lee OH, Chung HJ, Kim YS, Lee JI, Cho SG, Jeon YS, Lee KY, Ahn SI and Shin WY: Transarterial chemoembolization versus surgery/radiofrequency ablation for recurrent hepatocellular carcinoma with or without microvascular invasion. J Gastroenterol Hepatol 29(5): 1056-1064, 2014.

36 Wang K, Liu G, Li J, Yan Z, Xia Y, Wan X, Ji Y, Lau WY, Wu $M$ and Shen F: Early intrahepatic recurrence of hepatocellular carcinoma after hepatectomy treated with re-hepatectomy, ablation or chemoembolization: a prospective cohort study. Eur J Surg Oncol 41(2): 236-242, 2015.

37 Khor AY, Toh Y, Allen JC, Ng DC, Kao YH, Zhu G, Choo SP, Lo RH, Tay KH, Teo JY, Goh BK, Burgmans MC, Irani FG, Goh AS and Chow PK: Survival and pattern of tumor progression with yttrium-90 microsphere radioembolization in predominantly hepatitis B Asian patients with hepatocellular carcinoma. Hepatol Int 8(3): 395-404, 2014.

38 Bhangoo MS, Karnani DR, Hein PN, Giap H, Knowles H, Issa C, Steuterman S, Pockros P and Frenette C: Radioembolization with Yttrium-90 microspheres for patients with unresectable hepatocellular carcinoma. J Gastrointest Oncol 6(5): 469-478, 2015.

39 Shibata T, Isoda H, Hirokawa Y, Arizono S, Shimada K and Togashi K: Small hepatocellular carcinoma: is radiofrequency ablation combined with transcatheter arterial chemoembolization more effective than radiofrequency ablation alone for treatment? Radiology 252(3): 905-913, 2009.

40 Morimoto M, Numata K, Kondou M, Nozaki A, Morita S and Tanaka K: Midterm outcomes in patients with intermediate-sized hepatocellular carcinoma: a randomized controlled trial for determining the efficacy of radiofrequency ablation combined with transcatheter arterial chemoembolization. Cancer 116(23): 5452-5460, 2010.

41 Kim JH, Won HJ, Shin YM, Kim SH, Yoon HK, Sung KB and Kim PN: Medium-sized (3.1-5.0 cm) hepatocellular carcinoma: transarterial chemoembolization plus radiofrequency ablation versus radiofrequency ablation alone. Ann Surg Oncol 18(6): 1624-1629, 2011.

42 Kagawa T, Koizumi J, Kojima S, Nagata N, Numata M, Watanabe N, Watanabe T, Mine T and Tokai RFA Study Group: Transcatheter arterial chemoembolization plus radiofrequency ablation therapy for early stage hepatocellular carcinoma: comparison with surgical resection. Cancer 116(15): 3638-3644, 2010.

43 Kim JW, Shin SS, Kim JK, Choi SK, Heo SH, Lim HS, Hur YH, Cho CK, Jeong YY and Kang HK: Radiofrequency ablation combined with transcatheter arterial chemoembolization for the treatment of single hepatocellular carcinoma of 2 to $5 \mathrm{~cm}$ in diameter: comparison with surgical resection. Korean J Radiol 14(4): 626-635, 2013

44 Yin X, Zhang L, Wang YH, Zhang BH, Gan YH, Ge NL, Chen Y, Li LX and Ren ZG: Transcatheter arterial chemoembolization combined with radiofrequency ablation delays tumor progression and prolongs overall survival in patients with intermediate (BCLC B) hepatocellular carcinoma. BMC Cancer 14: 849, 2014. 
45 Mazzaferro V, Regalia E, Doci R, Andreola S, Pulvirenti A, Bozzetti F, Montalto F, Ammatuna M, Morabito A and Gennari L: Liver transplantation for the treatment of small hepatocellular carcinomas in patients with cirrhosis. N Engl J Med 334(11): 693-699, 1996.

46 Gomaa AI and Waked I: Recent advances in multidisciplinary management of hepatocellular carcinoma. World J Hepatol 7(4): 673-687, 2015.

47 Di Benedetto F, Tarantino G, Montalti R, Ballarin R, D'Amico G, Di Sandro S and Gerunda GE: Laparoscopic radiofrequency ablation in the caudate lobe for hepatocellular carcinoma before liver transplantation. J Laparoendosc Adv Surg Tech A 22(4): 400-402, 2012.

48 Colella G, Bottelli R, De Carlis L, Sansalone CV, Rondinara GF, Alberti A, Belli LS, Gelosa F, Iamoni GM, Rampoldi A, De Gasperi A, Corti A, Mazza E, Aseni P, Meroni A, Slim AO, Finzi M, Di Benedetto F, Manochehri F, Follini ML, Ideo G and Forti D.: Hepatocellular carcinoma: comparison between liver transplantation, resective surgery, ethanol injection, and chemoembolization. Transpl Int 11(Suppl 1): S193-196, 1998.

49 Wang YX, De Baere T, Idée JM and Ballet S: Transcatheter embolization therapy in liver cancer: an update of clinical evidences. Chin J Cancer Res 27(2): 96-121, 2015.

50 Montalti R, Mimmo A, Rompianesi G, Di Gregorio C, Serra V, Cautero N, Ballarin R, Spaggiari M, Tarantino G, D'Amico G, De Santis M, De Pietri L, Troisi RI, Gerunda GE and Di Benedetto F: Absence of viable HCC in the native liver is an independent protective factor of tumor recurrence after liver transplantation. Transplantation 97(2): 220-226, 2014.
51 Di Benedetto F, Tarantino G, Ercolani G, Baccarani U, Montalti R, De Ruvo N, Berretta M, Adani GL, Zanello M, Tavio M, Cautero N, Tirelli U, Pinna AD, Gerunda GE and Guaraldi G: Multicenter italian experience in liver transplantation for hepatocellular carcinoma in HIV-infected patients. Oncologist 18(5): 592-599, 2013.

52 Di Benedetto F, Tarantino G, Quintini C, Tirelli U and Berretta M, Hepatocellular carcinoma: beyond the boundaries of age. Anticancer Agents Med Chem 13(9): 1371-1377, 2013.

53 Valero V 3rd, Amini N, Spolverato G, Weiss MJ, Hirose K, Dagher NN, Wolfgang CL, Cameron AA, Philosophe B, Kamel IR and Pawlik TM: Sarcopenia adversely impacts postoperative complications following resection or transplantation in patients with primary liver tumors. J Gastrointest Surg 19(2): 272-281, 2015.

54 Nathan H, Segev DL, Bridges JF, Massie AB, Cameron AM, Hirose K, Schulick RD, Choti MA and Pawlik TM: Influence of nonclinical factors on choice of therapy for early hepatocellular carcinoma. Ann Surg Oncol 20(2): 448-456, 2013.

Received November 13, 2016

Revised January 3, 2017

Accepted January 17, 2017 